\title{
Collaborative cloud service model for delivering multimedia content in mCloud
}

\author{
Aleksandar Karadimce, Danco Davcev \\ Faculty of Computer Science and Engineering \\ University Ss Cyril and Methodius - Skopje \\ Skopje, Republic of Macedonia \\ akaradimce@ieee.org, danco.davcev@finki.ukim.mk
}

\begin{abstract}
Cloud environment is an efficient solution for both computing intensive and data intensive applications and it is a technology paradigm that is ideal for deploying a user centered mobile learning environment. Efficient use of cloud based services is a key component of successful task scheduling and resource allocation in cloud computing environment. In this paper, we propose a collaborative cloud service model for supporting the student's mobile learning process. The proposed collaborative model creates groups of students with joint interests collecting the relevant information from the existing social networks. The spirit of the proposed collaborative model comes from different aspects and influencing factors with respect to Quality of Experience (QoE) process. Experimental results gathered from using the OPNET simulator have verified the benefits of the proposed multimedia content adaptation collaborative model.
\end{abstract}

Keywords- content adaptation; collaborative model; collaborative cloud service; QoE; m-learning

\section{INTRODUCTION}

Today there is increased offer of different types of mobile electronic devices, in terms of their brand, their size (display dimensions), processing capabilities (single, double or quad core processors), storage capacity (different internal and external memory storage) and the mobile operating system. This represents challenge to mobile application developers to deliver applications and services that can be used on the variety of mobile devices, which are been released on the market every month. Considering the mobile device limitations in terms of data soring and capability of processing the solution is to utilize the new trend called mobile cloud computing (MCC) [1], which allows offloading and reducing the workload of mobile devices by exploiting the remote multimedia processing resources in the cloud.

The main benefit is to use a Service Oriented Architecture (SOA), which provides package functionality as a suite of interoperable services that can be used within multiple separate systems from several business domains [2]. The advantages of MCC and collaboration based on social networks are our motivation to propose the collaborative cloud service model for adaptive mobile learning environment.

Our previously proposed dynamically adapting system [3] has provided a unique effort to deliver the multimedia content in a mobile cloud computing learning environment (mCloud).
It provides the student with multimedia content that is tailored to his or her cognitive style and the content has been adapted according to the context-aware network conditions. After placing our system in the test case study, we realized that without considering the technical capabilities of the end user mobile devices, it is difficult to give efficient and appropriate estimation of the mobile learning system. Therefore, in this paper, we propose a collaborative cloud service model for supporting the student's mobile learning process in an attempt to find the optimal multimedia content distribution for various mobile learners. The proposed collaborative model will allow us to understand the relation between the different influencing factors, which are multi-dimensional constructs of perceptions and behaviors of a learner in multimedia learning system that have the biggest influence to the mobile user Quality of Experience (QoE).

The rest of the paper is organized as follows: in Section 2, we first give a brief overview of related work. In section 3, we provide architecture of collaborative cloud service model. In Section 4, we present the evaluation results of the proposed collaborative cloud service model for adaptive multimedia content delivery using simulation techniques. Finally, in Section 5 a conclusion and proposals for our future work are given.

\section{RELATED WORK}

Education based on the mobile cloud has been introduced as a novel state of the art education in the area of distance learning and there have been developed theoretical and analytical models for cloud-based learning to guide the design and development of an intelligent mobile cloud education system [4], [5], [6]. The mobile learning (m-learning) has been defined as any sort of leaning that happens when the learner is not at a fixed, pre-determined location or learning that happens when the learner takes advantage of learning opportunities offered by mobile technologies [7]. Based on the paper review, given by the Nagella and Govindarajulu in [7], the process of adaptation is achieved in terms of the learner context, the learner's knowledge levels, by the content that is been presented, the learner's style which may vary from learner to learner and adaptation to the device's context. This process leads to personalized learning content adaptation, which becomes increasingly important to meet the diverse needs imposed by devices, users, usage contexts, and infrastructure. The proposed Personalized Learning Content Adaptation 
Mechanism (PLCAM) is using variety of data mining techniques, including clustering and decision tree approaches, to efficiently manage a large number of historical learners' requests in order that the adaptation engines provide the adapted mobile educational content for the learners [5]. Some of the proposed analytical approaches to model the adaptive video streaming and delivery have provided a performance analysis which illustrates the trade-offs in service fairness and system efficiency and their model encompasses multiple-parent resource contribution in terms of bandwidth and CPU power [6]. The service providers that deliver content adaptation on a wide range of devices and networks, allow learners to receive improved user experience, with minimized storage and maintenance requirements on the server side.

Recently we have noticed the appearance of new protocols to cope with varying bandwidth, such as Apple's HTTP Live Streaming (HLS), Adobe with Flash-based Dynamic Streaming, Microsoft with Smooth Streaming for Silverlight and MPEG DASH (Dynamic Adaptive Streaming over HTTP) ISO standard [8]. Their research has showed that the increased segment duration can have negative effects of on video quality when using the UpstreamRR policy [8]. It also leads to increased start-up delay, slower convergence to the optimal quality and most importantly a significant increase in lag of streaming sessions compared to the live time [8]. With the growth of traffic produced by multimedia applications such as web and mobile TV, video on demand, personal video recording and targeted advertising services there is a challenge using different networks to deliver the multimedia content to a variety of user devices. There have been efforts to establish feedback fuzzy controller that supports dynamic and adaptive switching, by using the Network-Bandwidth-aware streaming version Switcher [9]. The proposed method is a lightweight method where it is possible to switch among different versions, such as high quality, medium quality, low quality, but it considers only the available bandwidth. The significance of the adaptive content delivery is a driver for new and competitive multimedia services that are based on cloud-computing infrastructures for end user entertainment services. The cloud is been utilized to offer the resources for content and semantic processing, for storage and delivery all of this to be provided in form of cloud services.

Therefore, the service oriented delivery will become homogeneous, ultimately leading to more speed and efficiency as well a higher level of accessibility and interactive user experience. The estimation of the student's perception for Quality of Experience provides analyses for the influence of cognitive style perception of different type of multimedia content, taking into consideration the multidimensional aspects of the QoE perception [10]. Creation of mobile learning content is not an easy task especially the process of delivering the multimedia content is highly dynamic and challenging. These processes add a new level of intelligence to both the mobile cloud-based environment and to the multimedia content assets, because it should be delivered for the consumer where he or she is located and the situational context.

With the development of technologies that will use intelligence in the way multimedia content is created, encoded, and manipulated, there is large plethora of networking applications for the mobile devices that will provide and enrich the user experience while consuming multimedia content [11]. The process of adaptation of multimedia content in m-learning systems is highly dependent from: learners' situation, educational needs and personal cognitive characteristics that should be considered in order to generate appropriately designed learning experience. Considering from different perspective, adaptations related to learning activities are referring to adaptation of the educational resources, tools and services that support the learning activities or adaptations to roles that participants have in the collaborative learning activities [12]. The collaborative filtering is based first on identifying the options and preferences of similar users in order to predict the preferences in order to recommend to others [13]. Authors Maria and Udo, in [13], have proposed interesting dialogue based on collaborative filtering model in order to improve the quality of the web search process.

\section{ARCHITECTURE OF COLLABORATIVE CLOUD SERVICE MODEL}

The collaborative cloud service model that we have proposed has to consider different influencing factors that affect the process of delivering multimedia content to the mobile learners. Major benefit of the proposed model is that it will join the objective - measurable influencing factors, such as bandwidth, jitter, delay and etc., with the subjective individual influencing factors in the m-learning system. The subjective factors that affect the multimedia content delivery have been measured using the QoE, which is a metric that quantifies these multifaceted, multidimensional factors that influence to the perceived quality. The accurate estimation of QoE on mobile services allows for control over the delivered multimedia content quality and an implementation of user centric management of these services [14].

In fact, this confirms with the QoE as the degree of delight or annoyance of a person whose experiencing involves an application, service, or system [15]. The process of estimating the QoE is conducted using the person's evaluation of the fulfillment of his or her expectations and needs with respect to the utility and/or enjoyment in the light of the person's context, personality and current state [15]. This means that the QoE is no longer an expression for user satisfaction of using a service, but it is a degree of delight or annoyance, which is more dynamic measure of personal experience. Therefore, in our work of defining a collaborative cloud service model for mlearning environment we are focusing more explicitly on the quality formation process and features that contribute to the perception of quality. Taking in consideration and analyzing all of the factors and parameters that affect the QoE, we have noticed different subjective factors that affect the immersive learning in cloud computing environment.

One of the subjective factors is the user's estimation for learning perception in m-learning systems, which includes not only users' enjoyment and satisfaction with a multimedia presentation, but also their ability for content perception. This helps during the process of creating learner's groups of interest using the user information of social networks. Group support formation provides relevant information for the process of estimating the learning perception in m-learning system. 
The second criterion that is important for modeling the adaptive multimedia content delivery system is the influence of the context-aware network conditions during the process of delivery of multimedia content [16]. The delivery of multimedia content in terms of network connectivity, primarily in connection with the high bandwidth, should provide appropriate high-definition multimedia content for the mobile learners. In circumstances when the learner experiences a poor network connection the system should provide multimedia content with a lower resolution or lower rate of frames per second for video and lower bit rate audio content [17].

The third criterion that influences the development of a collaborative cloud service model for adaptive multimedia content delivery should enable adaptive multimedia content according to the technical characteristics of the learner's device (mobile phone, tablet or PDA).

Given the complexity and multi-dimensional aspect of the QoE metrics, it is real challenging to provide appropriate model for the perception of quality that are based on cloud computing technology. The previously described factors that affect the process of delivery of multimedia contents for mobile learners will form the primary assertion of this research. This leads to the hypothesis that establishing a model that will perform adaptation of multimedia contents is going to contribute to higher quality and faster delivery of relevant contents for the learners.

The architecture of collaborative cloud service model that we propose is given in Figure 1. The mobile cloud computing environment offers to mobile learners to use heterogeneous types of connections to Internet, such as WiFi, WiMAX, UMTS, GPRS, HSDPA and EDGE. The architecture considers the influence of the social network groups of interest, and together they support the group support formation process. The mobile learner is connected to the system for m-learning using his mobile device. The cloud broker is responsible for authenticating the user to the m-learning system and then for processing the user requests for multimedia content. After receiving the user request the mobile cloud is responsible to process the request through a series of three services.

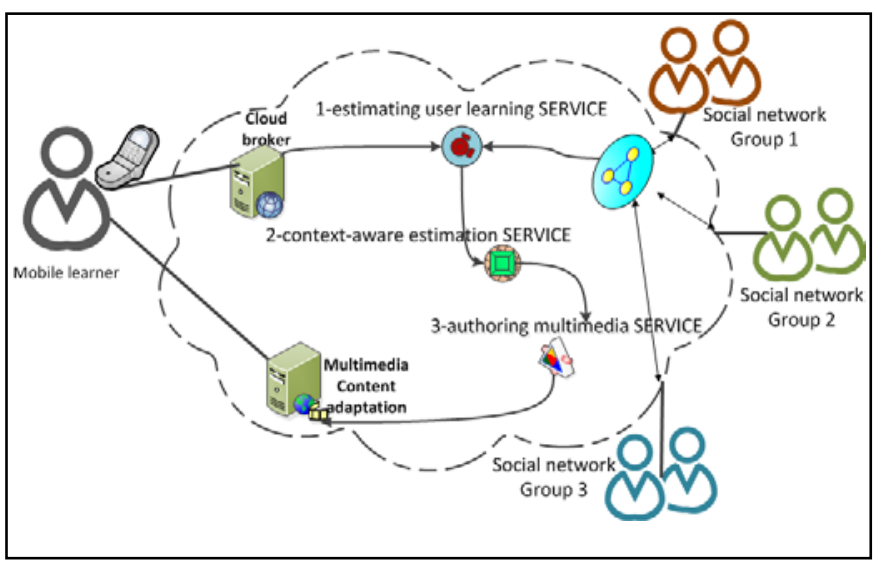

Figure 1. Architecture of collaborative cloud service model.

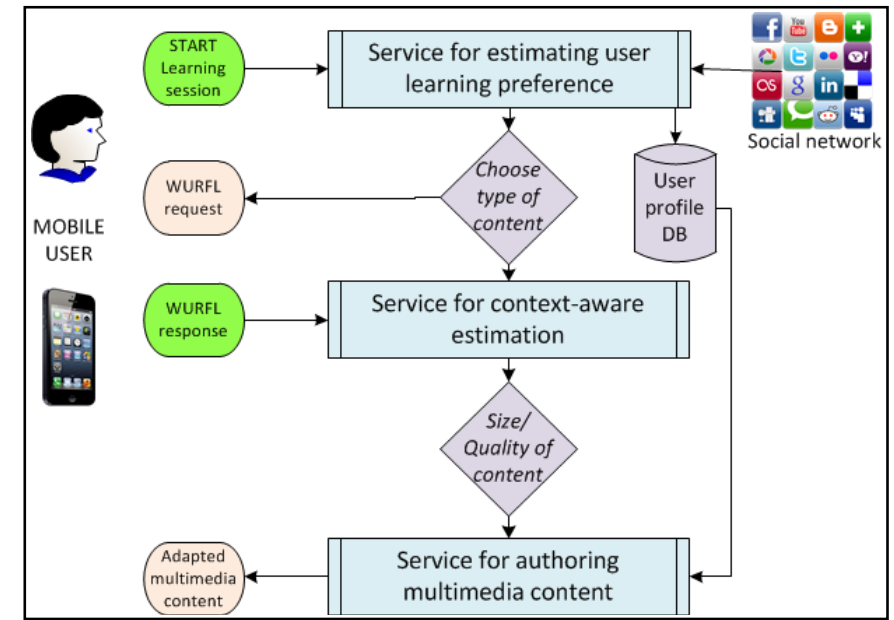

Figure 2. Interaction diagram for collaborative cloud service model.

The first service is estimating the user learning preference using the information from different social network groups of interest. The next service is adapting the multimedia content according to the context-aware conditions of the mobile learner. The last step is to transcode the multimedia content in order to be compatible for the mobile device. The authoring multimedia service will complete the learning content that should be delivered to the mobile learner. Multimedia content adaptation server located in the mobile cloud will provide delivery of the adapted multimedia content to the leaner. In connection with this architecture, we present on Figure 2 the interaction- functional diagram that clarifies the collaborative multimedia content delivery model.

After successful authentication to the mCloud, the users can start learning session. In order to estimate the user learning preferences we will use the information gathered from the interest groups of its social networks. Next, we have to discover the learners' mobile device operating system (mobile OS) and supported media format types and supported encoding preferences.

Possible solution is to use the WURFL, the Wireless Universal Resource FiLe, which is a software component that maps HTTP Request headers to the profile of the HTTP client (Desktop, Mobile Device, Tablet, etc.) that issued the request [18]. This software component is able to detect learner's mobile device technical capabilities during the initial HTTP request and compare it with existing devices from device description repository (DDR) [19].

Using the information gathered by the WURFL component we will be able to make collaborative adaptation of the multimedia content to the mobile device supported capabilities (device type, browser or screen size). This way the WURFL model of detection is appropriate for discovering the technical capabilities of mobile device and will contribute to the authoring service of this framework. Last service for authoring the multimedia content provides adapted multimedia for the mobile learner. 


\section{EVALUATION OF THE COLLABORATIVE CLOUD SERVICE MODEL FOR ADAPTIVE MULTIMEDIA CONTENT DELIVERY}

The collaborative cloud service model for adaptive multimedia content delivery has been demonstrated using a simulation tool called OPNET Modeler. This software product, released by OPNET Technologies, is used to support the research process by providing easy analysis and design of communication networks, application, protocols, and devices [20]. According to experts [20], OPNET Modeler offers the fastest discreet event simulation engine when compared with other networking solutions in the industry. The objective of the simulation is to have a model that maximizes the network performance, taking into consideration the context-aware constraints and the required services to be offered to different types of users. The model is consisted of server and network of interconnected mobile clients and has been implemented in OPNET Modeler using two different scenarios.

The first scenario considers that the server is used for delivering multimedia content to multiple mobile clients without using any caching or adaptation mechanism. The results from the first scenario from the simulation are represented with red line. The second scenario provides the collaborative cloud service model of adaptive multimedia content delivery in mCloud to practice the benefits of multimedia content adaptation. This has been accomplished by transcoding of multimedia content that has been delivered to the mobile clients. The results from the adaptive scenario have been marked with blue color. Therefore, we have gathered the global statistics for the response time, network bandwidth and http traffic received. The simulation was done using the following hardware specification: Server with CPU (Intel i52500 with $3.30 \mathrm{GHz}$ ) and $4 \mathrm{~GB}$ RAM, which uses 64-bit Operating system. We have defined simulation to run 6 hours of multimedia content delivery.

This research includes analysis of global statistics based on simulation results, both from clients and server side. The gathered results from mobile client's http object response time in average provides better results for the adaptive scenario, see Figure 3. The object response time for the adaptive delivery scenario, which is marked with blue color in Figure 3 , has better start-up object delivery in the first half-hour of collaborative m-learning multimedia content delivery process.

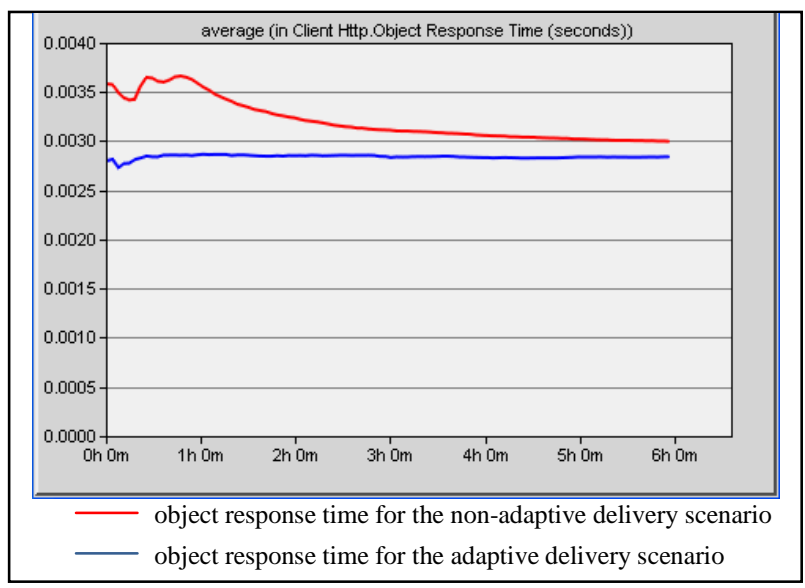

Figure 3. Average HTTP client object response time (seconds).

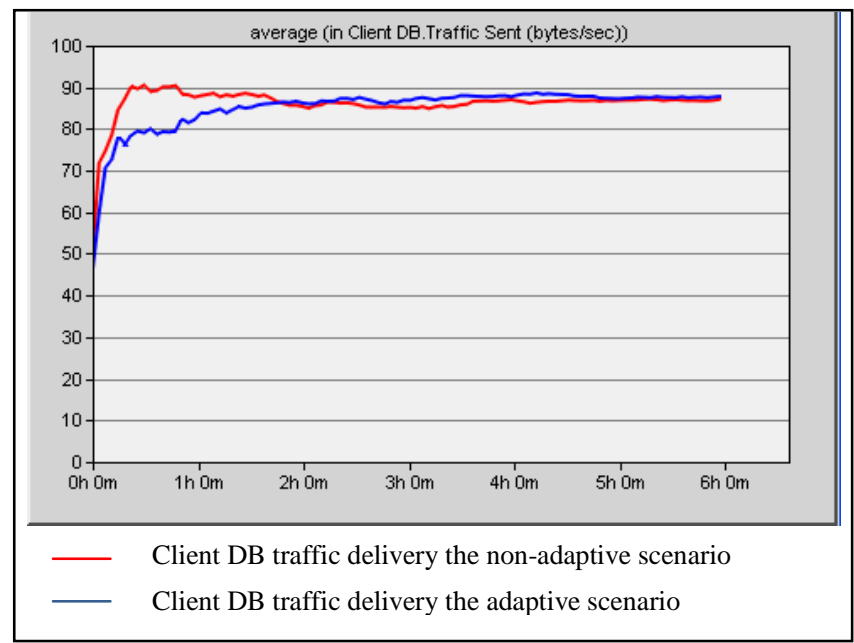

Figure 4. The average Client DB traffic (bytes/sec).

Next, the subject of our analysis was the database traffic between the server and clients. The gathered simulation results from the client's database traffic sent in average are given in Figure 4. These results provide better insight to understand the benefit of using scenario with collaborative adaptive multimedia content delivery, rather than the scenario without any adaptation. The improvement of DB traffic delivery is mostly visible in the first hour of running the simulation. The peak of DB traffic is reached by 90 bytes/sec in the first minutes in the non-adaptive scenario, while the second scenario (the adaptive), whose results have been marked with blue color, is using only 80 bytes/sec in the first minutes of the simulation. After the second hour of the simulation we can notice certain alignment in client DB traffic for the both scenarios.

The comparison of the average server FTP traffic showed the overlapped simulation results for both scenarios, adaptive and non-adaptive multimedia content delivery, devoted to the server FTP traffic received. We can conclude that there is not significant impact on the server side, except for the slide increase of traffic in the first two hours of simulation, see Figure 5. These simulation results confirm that the collaborative adaptive approach of delivery of multimedia content will not experience significant impact on the traffic delivery compared with the regular content delivery in first scenario. The next simulation results provide the global statistics for HTTP object response time and FTP upload response time. These statistics provide general information regarding a specific parameter. Significant difference can be observed between both scenarios if we analyze the global statistics on FTP upload response time, see Figure 6. 


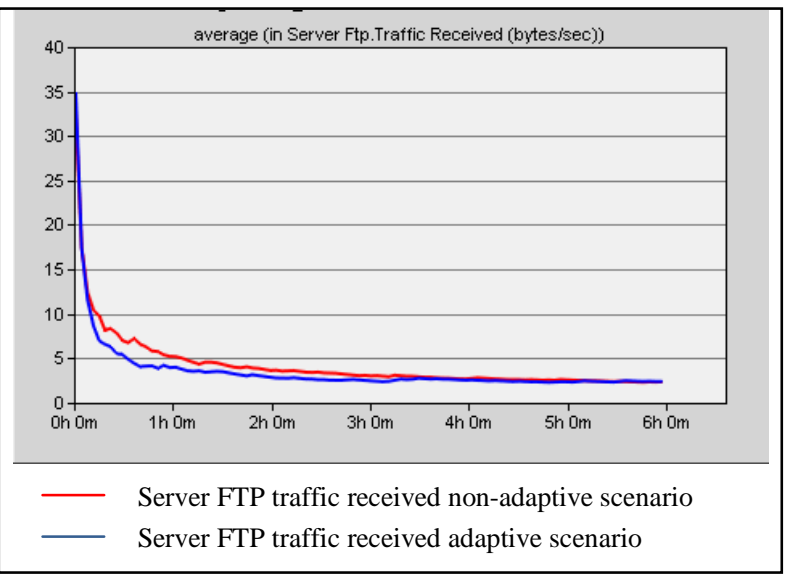

Figure 5. The average Server FTP traffic (bytes/sec).

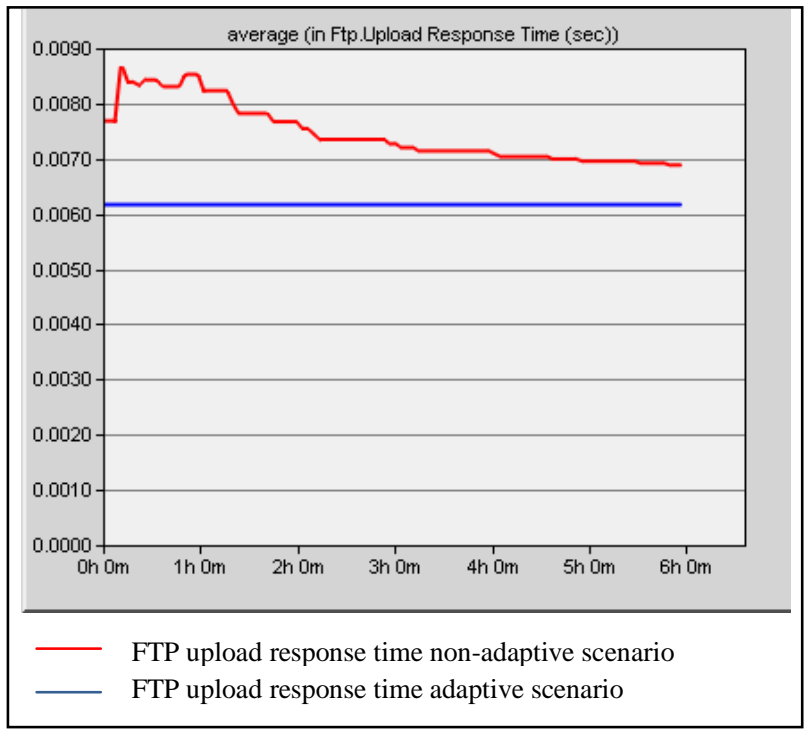

Figure 6. The average FTP upload response time (seconds).

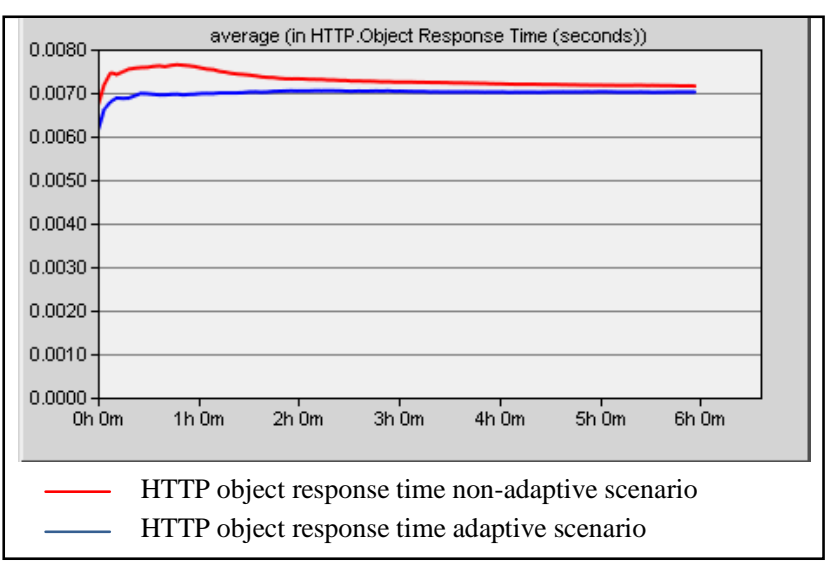

Figure 7. The average HTTP object response time (seconds).

The adaptive scenario is generating substantially better upload response time for the multimedia content delivery for the clients, marked with blue line, compared to the nonadaptive scenario marked with red line. Another difference between the two scenarios can be noticed when analyzing the results from the HTTP object response time. The average
HTTP object response time is higher for the non-adaptive scenario; this difference is especially visible at the beginning of the simulation, see Figure 7. All of the previously analyzed parameters confirm and approve the superiority of the collaborative cloud service model for adaptive multimedia content delivery in mCloud.

\section{CONCLUSION AND FUTURE WORK}

The mobile cloud computing role is to offload and to reduce the workload of mobile devices by exploiting the remote multimedia processing resources in the cloud. The proposed collaborative cloud service model for delivering multimedia content in mCloud has been discussed for the purpose of m-learning. It is based on the SOA architecture that uses different services to provide adaptation of the multimedia content that should be delivered to the user's mobile devices. Our simulation results confirm the benefits of the multimedia content adaptation.

The proposed collaborative cloud service model in combination with additional similar services can be easily applied and used in m-health, m-commerce, m-social services and other rising mobile perspectives. An autonomous and robust selection and scheduling of such services from a pool of available services, is the subject of our future work.

\section{REFERENCES}

[1] P. Mell and T. Gance, "The NIST Definition of Cloud Computing (Final)," NIST Special Publication, pp. 800-145, Sept. 2011. <http://csrc.nist.gov/publications/nistpubs/800-145/SP800-145.pdf > 2014.02.03

[2] M. P. Papazoglou and Willem-Jan Heuvel. "Service oriented architectures: approaches, technologies and research issues," The VLDB Journal 16, 3 (July 2007), pp. 389-415, 2007, DOI=10.1007/s00778007-0044-3.

[3] A. Karadimce and D. Davcev, "Adaptive Multimedia Learning Delivered in Mobile Cloud Computing Environment," in The Fourth International Conference on Cloud Computing, GRIDs, and Virtualization, IARIA CLOUD COMPUTING 2013, 27-31 May 2013, Valencia, Spain, pp. 62-67, ISBN: 978-1-61208-271-4.

[4] R. S. Khune and J. Thangakumar, "A Cloud-Based Intrusion Detection System for Android Smartphones," in 2012 International Conference on Radar, Communication and Computing (ICRCC), December 2012, pp. 180-184, ISBN: 978-1-4673-2756-5.

[5] J. M. Su, S. S. Tseng, H. Y. Lin, and C. H. Chen, "A personalized learning content adaptation mechanism to meet diverse user needs in mobile learning environments," User Modeling and User-Adapted Interaction 21, 1-2 (April 2011), pp. 5-49, DOI=10.1007/s11257-0109094-0, <http://dx.doi.org/10.1007/s11257-010-9094-0 > 2014.02.07

[6] R. Iqbal and S. Shirmohammadi, "An analytical approach to model adaptive video streaming and delivery," in Proceedings of the 2010 ACM workshop on Advanced video streaming techniques for peer-topeer networks and social networking (AVSTP2P '10), ACM, New York, NY, USA, pp. 55-58, DOI $=10.1145 / 1877891.1877905$, $<$ http://doi.acm.org/10.1145/1877891.1877905 > 2014.02.07

[7] U. B. Nagella and D. P. Govindarajulu, "Adaptive Approaches to Context Aware Mobile Learning Applications," International Journal of Computer Science and Security, Volume (2): Issue (2), 2009, ISSN:1985-1553.

[8] J. Famaey, S. Latré, R. Brandenburg, M. O. Deventer, and F. Turck, "On the Impact of Redirection on HTTP Adaptive Streaming Services in Federated CDNs," in the International Conference on Autonomous Infrastructure, Management and Security, Lecture Notes in Computer Science (AIMS - 2013), vol. 7943, pp. 13-24, ISSN: 0302-9743. 
[9] K. De Moor and L. De Marez, "The Challenge of User- and QoE-centric research and product development in today's ICT-environment," Published in J. Pierson, Mante-Meijer, E., Loos, E. and Sapio, B. (Eds.) Innovating for and by users, Luxembourg: Office for Official Publications of the European Communities, 2008, pp. 77-90.

[10] Pengcheng Xiong, Jialie Shen, Qingyang Wang, Deepal Jayasinghe, Jack Li and Calton Pu, "NBS: A Network-Bandwidth-Aware Streaming Version Switcher for Mobile Streaming Applications under Fuzzy Logic Control”, In Proc. of IEEE International Conference On Mobile Services (MS'12), Honolulu, Hawaii, USA, June. 2012.

[11] A. M. Arsenio, "Intelligent Approaches for Adaptation and Distribution of Personalized Multimedia Content," in Intelligent Multimedia Technologies for Networking Applications: Techniques and Tools by Dimitris N. Kanellopoulos, 2013, ISBN: 9781466628335, pp. 197-224.

[12] S. Gomez, P. Zervas, D. G. Sampson, and R. Fabregat, "Delivering Adaptive and Context-Aware Educational Scenarios via Mobile Devices," Advanced Learning Technologies (ICALT), pp. 197-201, 2012 IEEE 12th International Conference on Advanced Learning Technologies, 2012.

[13] M. Fasli and U. Kruschwitz, "Applying collaborative filtering ideas to improve a dialogue system for Web search", in Proceedings of International Conference of Intelligent Agents, Web Technology and Interenrt Commerce - IAWTIC'2011, Las Vegas.
[14] V. Menkovski and A. Liotta, "QoE for mobile streaming," Published in Mobile Multimedia - User and Technology Perspectives, InTech Open Access Publisher, ISBN: 978-953-307-908-0, 2012.

[15] A. Raake and S. Egger: Quality and Quality of Experience. In S. Möller and A. Raake, editors, Quality of Experience: Advanced Concepts, Applications and Methods. Springer, 2014.

[16] H. J. La and S. D. Kim, "A Conceptual Framework for Provisioning Context-aware Mobile Cloud Services," in IEEE CLOUD, pp. 466-473, IEEE, ISBN: 978-1-4244-8207-8.

[17] K. Miller, E. Quacchio, G. Gennari, and A. Wolisz, "Adaptation Algorithm for Adaptive Streaming over HTTP," in Proc. of 19th International Packet Video Workshop, ISBN:978-1-4673-0299-9.

[18] D. Seo and I. Jung: Network-adaptive autonomic transcoding algorithm for seamless streaming media service of mobile clients. Multimedia Tools Appl. 51, 3, pp. 897-912, DOI: 10.1007/s11042-009-0421-6 (2011)

[19] WURFL - Wireless Universal Resource FiLe, a Device Description Repository (DDR) by ScientaMobile. http://wurfl.sourceforge.net/ <accessed: 21.06.2014>

[20] Z. Lu and H.Yang, "Unlocking the Power of OPNET Modeler," Cambridge: Cambridge University Press, ISBN: 9780521198745, 2012, <http://hdl.handle.net/2086/7137 > <accessed: 25.05.2014> 